\title{
Efficacy of Chemotherapy for Malignant Pleural Mesothelioma According to Histology
}

\section{Susana Cedres ( $\square$ scedres@vhio.net)}

Vall d'Hebron Hospital Universitari

Juan David Assaf

Vall d'Hebron Hospital Universitari

\section{Patricia Iranzo}

Vall d'Hebron Hospital Universitari

\section{Ana Callejo}

Vall d'Hebron Hospital Universitari

Nuria Pardo

Vall d'Hebron Hospital Universitari

\section{Alejandro Navarro}

Vall d'Hebron Hospital Universitari

Alex Martinez-Marti

Vall d'Hebron Hospital Universitari

David Marmolejo

Vall d'Hebron Hospital Universitari

\section{Alejandra Rezqallah}

Vall d'Hebron Hospital Universitari

\section{Caterina Carbonell}

Vall d'Hebron Hospital Universitari

Joan Frigola

Vall d'Hebron Hospital Universitari

\section{Ramon Amat}

Vall d'Hebron Hospital Universitari

\section{Anna Pedrola}

Vall d'Hebron Hospital Universitari

\section{Rodrigo Dienstmann}

Vall d'Hebron Hospital Universitari

Enriqueta Felip

Vall d'Hebron Hospital Universitari

\section{Research Article}


Keywords: malignant mesothelioma, immunotherapy, epithelioid, histology

Posted Date: July 9th, 2021

DOI: https://doi.org/10.21203/rs.3.rs-677246/v1

License: (c) (1) This work is licensed under a Creative Commons Attribution 4.0 International License. Read Full License 


\section{Abstract}

\section{Purpose}

CheckMate 743 trial demonstrated survival benefit of immunotherapy in first line in MPM with some differences in the efficacy of chemotherapy according to histology. The objective of this study is to characterize the impact of chemotherapy according to histology in patients diagnosed with MPM at our institution.

\section{Methods}

Clinical records of all MPM patients diagnosed at Vall d'Hebron University Hospital between November 2002 and April 2020 were reviewed. Associations between clinical variables and outcomes were assessed with Cox regression models. Survival data were calculated by the Kaplan-Meier method.

\section{Results}

189 patients were included with $76 \%$ of tumors classified as epithelioid subtype. First line chemotherapy was offered to $85 \%$ of patients. Median survival in overall population was 21.3 months ( $95 \% \mathrm{Cl} 17.2-24.3)$. We found that patients with epithelioid tumors had better overall survival (OS) and progression free survival (PFS). Median OS of epithelioid patients treated with first line chemotherapy was 26.7 months versus 15.0 months in nonepithelioid patients (HR2.25 Cl95\% 1.4-3.4; $\mathrm{p}<0.001)$. Median PFS for patients with epithelioid tumors treated with chemotherapy was 4.8 months versus 3.6 months in nonepithelioid (HR1.5 Cl95\% 1.0-2.3; p=0.03). The improvement of outcomes in patients with epithelioid histology was detected in patients treated with cisplatin or carboplatin. Histology was not a predictive factor for the platinum agent sensitivity ( $p$ of interaction PFS $=0.09$, $p$ of interaction $0 S=0.65$ ).

\section{Conclusions}

In our series, patients with nonepithelioid tumors presented worse prognosis. Although epithelioid tumors exposed to cisplatin had higher PFS, histology was not a clear predictor of chemotherapy efficacy.

\section{Introduction}

Malignant pleural mesothelioma (MPM) is a rare and aggressive cancer arising from the mesothelial cells lining the pleura. Asbestos exposure is the major risk factor for mesothelioma with a very prolonged latency period between exposure to asbestos and the development of mesothelioma (20-50 years) [1]. The incidence rates of mesothelioma in the United States are 0.9 for men and 0.3 for women and in Europe 1.7 for males and 0.4 for females per 100000 habitants [2]. The annual incidence of mesothelioma is increasing in Great Britain and Australia and it is predicted to increase in countries with poor regulation of asbestos mining. 
Malignant mesothelioma is often refractory to standard chemotherapy regimens and exhibit poor prognosis, with overall survival being on the order of 9-18 months after diagnosis [3, 4]. ECOG performance status, stage and histology are the strongest prognostic factors among patients with mesothelioma. The World Health Organization (WHO) classification includes three main histological subtypes (epithelioid, sarcomatoid and biphasic) with a different prognosis. Epithelioid histology is associated with a more favorable prognosis.

Treatment options for MPM patients who are not eligible for surgery are very limited. Platinum-based chemotherapy combined with an antifolate lead to a median survival of about 12-16 months [3]. The addition of bevacizumab or Tumor-Treating fields (TTF) to chemotherapy increases survival (18.8 and 18.2 months respectively) $[4,5]$. Carboplatin in association with pemetrexed is an accepted alternative option for patients who may not tolerate cisplatin [6, 7].

There remains an unmet clinical need for new, effective therapies that can improve outcomes in the first line. In recent years a dramatic improvement in advanced cancers therapy has been achieved with immune checkpoint blockade. However, results of early studies with immunotherapy in mesothelioma are contradictory, and currently only Japan has approved nivolumab in second line setting and US the combination of nivolumab plus ipilimumab in first line setting. Initial studies using single drug checkpoint inhibitors in previously treated patients demonstrated some efficacy with a median progression free survival (PFS) between 2-6 months with nivolumab and about 5 months with pembrolizumab [8-10]. However, the results of randomized trial are controversial [11-13]. Pembrolizumab and tremelimumab failed to show improvement in PFS and overall survival (OS) in second-third line versus chemotherapy or placebo, but recently the CONFIRM trial demonstrated that nivolumab improved PFS and OS versus placebo in relapsed MPM [13]. The combination of immune checkpoint inhibition with ipilimumab and nivolumab in previously treated patients demonstrated similar results for the combination and monotherapy in the MAPS2 trial, and in first line the combination demonstrated being superior to chemotherapy in the pivotal CheckMate 743 in terms of survival (OS 18.1 months) leading to the approval of this combination by FDA $[14,15]$. In a preplanned subanalysis considering histology, improvements in efficacy of immunotherapy over chemotherapy were statistically significant among those with nonepithelioid histology but not for epithelioid histology. Authors suggested that the differences were due to reduced efficacy of chemotherapy in nonepithelioid MPM. However, the pivotal trial INITIATE which led to the approval of cisplatin plus pemetrexed in MPM did not evaluated the efficacy of chemotherapy by histology [3].

The aim of our study is to evaluate the efficacy of the chemotherapy by histological subgroups in a realworld series of MPM patients.

\section{Methods}

\subsection{Study design}


This retrospective cohort study used real-world data from the electronic medical records from the Vall d 'Hebron Hospital Universitari to identify patients with MPM who had initiated systemic therapy under routine clinical practice between February 2002 to February 2020. The study selection period encompasses the dates when immunotherapy was been evaluated in MPM, but not approved. Patients were followed longitudinally until death or their last visit prior to data cutoff. Demographic information, asbestos exposure, stage at initial diagnosis, sites of metastases, cancer treatment, medical history, disease characteristics and data on tumor evaluation (including progression of the disease and response to treatment), were considered as appropriate. This study was approved by local Ethical Committee at Vall d'Hebron Hospital Universitari. Informed consent form was waived due to the retrospective nature of the study and permission for data usage was obtained from the local ethic committee (Ethical Committee at Vall d'Hebron Hospital Universitari). All methods were performed in accordance with the relevant guidelines and regulations

\subsection{Patients}

The study cohort included patients with confirmed MPM who had received at least one line of therapy for their disease between February 2002 to February 2020, had clinical record available and were 18 years or older. To allow for sufficient follow-up for clinical outcomes, patients entered the cohort no later than 10 months prior to data cutoff (March 2021). One hundred eighty-nine consecutive cases of MPM were retrospectively collected. Clinicopathologic information gathered included complete history, age, sex, performance status (PS), asbestos exposure, tumor stage and histological subtype. Neutrophil-tolymphocyte ratio was calculated as the ratio between neutrophils and lymphocyte in the blood analysis obtained at the time of diagnosis. The tumor stage was defined according to the International Union Against Cancer's tumor-node metastasis $8^{\text {th }}$ classification and sub-classified histologically according to the WHO guidelines $[16,17]$. All cases were reviewed by the local pathologist with expertise in the diagnosis of MPM. All tumor biopsies analyzed were obtained by surgery (147 patients) or core needle biopsy (42 patients).

\subsection{Study outcomes}

The primary objective of this study was to describe the association of the histology with overall survival (OS) and progression-free survival (PFS) in MPM who received systemic chemotherapy. Secondary analyses included assessment of the outcomes in patients treated with immunotherapy and a study of prognostic factors in a real-world series of MPM. OS for each patient was defined as the time to death from diagnosis of malignancy. Progression of the disease was determined by physician assessment based on radiographic evidence. PFS was defined as the time until the earliest record of actual disease progression or death from any cause from initiation of line therapy. PFS was analyzed by therapy type.

\subsection{Statistical analysis}

Data were censored at last follow up for patients without relapse or death. Median follow-up time was calculated with reverse Kaplan-Meier estimator [18]. Median follow-up time was calculated with reverse 
Kaplan-Meier estimator. OS was calculated from diagnosis of malignancy until death due to any cause or until the date of last follow-up visit for still alive patients. Survival analysis that compared efficacy of chemotherapy by histology was carried out using the Kaplan-Meier curves and the significance was verified by a log-rank test. All $p$ values were determined by two-sided tests and $p$ values $<0.05$ were considered significant. Multivariable analysis was done using the Cox regression model including only the clinical variables that showed significance in univariable analysis. A model with interaction between histology and platinum agent was constructed to determine whether the predictive value of chemotherapy agent is dependent on histology. Data analysis was produced by the R statistical software version 4.0

\section{Results}

\subsection{Patient population}

We studied 189 patients with MPM whose clinicopathologic characteristics are summarized in Table 1. The median age was 68 years (range $45-88)$. Patients were predominantly male (70\%), smokers $(50 \%)$, had previous asbestos exposure (75\%) and stage III (45\%). The median neutrophil-lymphocyte ratio (NLR) was 5.2 and $58 \%$ have NLR less than 5 . The total cohort comprised 145 epithelioid tumors, 17 sarcomatoid, 14 biphasic, and 13 cases with histological type not specified.

Out of the entire group, none of the patients was considered for extrapleural pneumonectomy and 161 patients (85\%) were treated with chemotherapy. Regarding the type of systemic treatment, 134 patients (84\%) received platinum plus pemetrexed in first line. Among them 102 patients received cisplatin plus pemetrexed and 32 patients received carboplatin plus pemetrexed. Additionally, 16 patients (10\%) were included in clinical trials in first line. The median number of cycles of chemotherapy in first line was 5 for patients treated with cisplatin or carboplatin. The objective response rate (ORR) to chemotherapy was $38 \%$ in epithelioid and $22 \%$ in nonepithelioid tumors with similar efficacy of chemotherapy in epithelioid patients treated with cisplatin or carboplatin.

\subsection{Survival analysis}

Median survival of the entire group was 21.3 months $(95 \% \mathrm{Cl} 17.2-24.3$ months). There was an improved survival rate in patients with good PS, epithelioid subtype histology, stage II and NLR (Figure 1). Median survival for patients with PSO, 1 and 2 was 28.8 months, 18.8 months and 2.4 months respectively $(p<0.001)$. Median survival for patients with stage II was 28 months versus 18.4 months for patients with stage III/IV ( $p=0.019 \mathrm{Cl} 95 \% 1.2-2.5)$. Patients with epithelioid histology had a median survival of 21.3 months versus 9.6 months in nonepithelioid patients (HR 2.4, CI95\% 1.6-3.4, $\mathrm{p}<0.001$ ). Median survival for patients with NLR $<5$ was 25.1 months versus 12 months for patients with NLR>5 (HR1.82, CI95\% 1.3$2.6, p<0.001)$. We did not find differences in survival according to gender, smoking and asbestos exposure ( $p>0.05)$. Median OS in patients which received first line systemic therapy was 21.6 months $(19.1-25.2)$. 


\subsection{Survival and type of treatment}

We assess the magnitude of the treatment effect considering the type of treatment received. Median survival of patients treated in first line with cisplatin plus pemetrexed was 23.1 months versus 16.4 months for patients treated with carboplatin plus pemetrexed (HR 0.4, Cl95\% 0.3-0.7, p<0.001), Figure 2. In second line, patients treated with cisplatin plus pemetrexed had a median OS of 43.7 months versus 18.5 months for patients treated with carboplatin plus pemetrexed (HR 0.5, Cl95\% 0.1-1.9, p=0.32). Median OS in second line was 17.1 months for patients who received platinum versus 10.7 months for patients treated without platinum agent (HR 0.5, Cl95\% 0.3-0.8, p=0.008).

In total 27 patients received cancer immunotherapy in second or further line (14 patients with anti-CTLA4, 8 patients with antiPD-1/PD-L1, 4 patients with anti-mesothelin and 1 patient with oncolytic virus). Median OS for patients treated with cancer immunotherapy was 26.4 months versus 20.9 months for patients that did not receive immunotherapy (HR 0.84, Cl95\%0.5-1.3, p=0.46).

\subsection{Survival and histology}

In our series, patients with epithelioid subtype presented a median survival of 24.3 months versus 9.6 months biphasic, 8.6 months sarcomatoid and 20 months in no other specified histology $(p<0.001)$. When we analyzed the survival of patients who received first line chemotherapy according to histology, we found that patients with epithelioid tumors had better survival (Figure 3). Median OS for patients with epithelioid tumors treated with chemotherapy in first line was 26.7 months versus 15.0 months in patients with nonepithelioid tumors (HR2.25 Cl95\% 1.4-3.4; $\mathrm{p}<0.001)$. Analyzing all the histologies, median OS for patients treated with first line chemotherapy was 26.7 months in epithelioid, 11.2 months in biphasic, 10.7 months in sarcomatoid and 22 months in no other specified histology. Moreover, the PFS was also better for patients with epithelioid histology treated with first line chemotherapy (PFS 4.8 months versus 3.6 months in epithelioid and nonepithelioid patients respectively (HR1.5 Cl95\% 1.0-2.3; $p=0.03$ ). Then we analyzed if the differences in survival according to histology were due to the type of systemic treatment received. The median OS for epithelioid patients receiving cisplatin plus pemetrexed was 30.7 months versus 17.2 months for nonepithelioid (HR2.7 Cl95\% 1.6-4.5; $\mathrm{P}<0.001$ ). For patients who received carboplatin plus pemetrexed in first line the median OS was 26.7 months in epithelioid versus 14.8 months in nonepithelioid patients (HR2.7 CI95\% 1.3-5.8; $p=0.008$ ). Median PFS was numerically higher in patients with epithelioid tumors who received cisplatin plus pemetrexed versus nonepithelioid population (5.1 months versus 3.6 months; HR 1.4 CI95\% 0.91-2.3; $p=0.11$ ). Similarly, patients with epithelioid tumors treated with carboplatin has median PFS 4.5 months versus 3.6 moths in patients with nonepithelioid MPM (HR 1.99 Cl95\% 0.96-4.1; $p=0.06$ ). Despite the worse prognosis for nonepithelioid MPM, the interaction test with Cox regression model did not show significant value of histology as a predictive factor for the platinum agent sensitivity (OS, p interation $=0.65$, PFS $p$ interaction $=0.09$ ). (table 2)

\subsection{Survival and cancer immunotherapy}


Finally, we assess the impact of histology for patients treated with cancer immunotherapy. In total 27 patients were treated with cancer immunotherapy in second and further lines. Median OS for these patients treated with immunotherapy was 28.3 months for epithelioid versus 13.8 months for nonepithelioid patients (HR $3.4 \mathrm{Cl} 95 \%$ 1.3-8.7; $\mathrm{p}=0.01$ ). We did not detect difference in the PFS of patients treated with cancer immunotherapy in second line according to histology ( 2.7 months versus 3 months in nonepithelioid (HR 0.7 Cl95\% 0.2-1.7; p=0.43). (Figure 4)

\subsection{Multivariable analysis}

Using multivariable analysis with a Cox regression model that included significant variables in the univariate model, we found that epithelioid histology, NLR and treatment with cisplatin versus carboplatin remain significant prognostic factor for survival.

\section{Discussion}

The aim of this study is to investigate the impact of histology in the efficacy of systemic treatment in a real-world database MPM. Our results show that epithelioid tumors presented better outcomes when received chemotherapy, irrespective of the platinum agent.

Histology has been broadly studied in MPM and well recognized as a prognostic factor $[19,20]$. The two prognostic scoring systems classically used in mesothelioma were developed previous to the routine use of pemetrexed and both scores included nonepithelial histology as predictor of poor survival (EORTC and CALGB) with a difference in median survival around 3 months between epithelioid and nonepithelioid. However, the role of histology as predictive factor of efficacy to treatment with chemotherapy in advanced MPM has not been well address in the clinical trials. In the era of pemetrexed treatment, only a retrospective analysis of 131 patients treated with chemotherapy demonstrated that epithelioid histology was assessed with clinical benefit from first line chemotherapy [21].

Recently, the study CheckMate 743 comparing chemotherapy versus nivolumab plus ipilimumab in untreated patients demonstrated that the combination of immunotherapy was superior in terms of survival [15]. In the trial patients were stratified by histology (epithelioid versus nonepithelioid) including $75 \%$ of patients with epithelioid histology. In a preplanned subanalysis, the improvement of immunotherapy over chemotherapy was clearly superior in nonepithelioid patients with a median OS of 18.1 months with immunotherapy and 8.8 months with chemotherapy. For the overall population no differences in terms of PFS were detected. Authors concluded that survival benefit with nivolumab plus ipilimumab over chemotherapy was observed regardless of histology with better outcomes for chemotherapy in epithelioid histology. The presence of PD-L1 also predicted improvement with nivolumab plus ipilimumab over chemotherapy, but according to the authors of the study, PD-L1 results were descriptive only, precluding definitive conclusions.

The pivotal trial EMPHACIS published by Vogelzang demonstrated that combination of therapy with cisplatin plus pemetrexed was superior to cisplatin alone [3]. In the trial $68 \%$ of patients were epithelioid 
histology, but no data about the efficacy by histological subgroups were reported. Similarly, in a subsequent phase IV trial evaluating the role of pemetrexed in mesothelioma, the efficacy by histology was not reported [22]. Raltitrexed, the other antimetabolite approved in malignant mesothelioma also demonstrated improved in survival in combination with cisplatin and about two thirds of patients were epithelioid [23]. In this trial patients with nonepithelial tumors presented worse prognostic in univariate and multivariable analysis, but no data were published regarding the predictive role of the treatment with histology. Carboplatin in association with pemetrexed is an alternative option for patients who may not tolerate cisplatin [6, 7]. In the larger of two randomized phase II studies response was observed in patients with epithelial or mixed histology, but no response was registered in patients with sarcomatoid mesothelioma.

In order to improve the outcomes of the chemotherapy, the addition of antiangiogenics or TTFields has been explored. The MAPS trial demonstrated that survival was significantly extended with the addition of bevacizumab to chemotherapy [4]. In a preplanned subgroup analyses, the effect on survival of the bevacizumab containing regimen compared with standard chemotherapy was homogeneous when the analysis was stratified by histology subtype, moreover, the effect was more pronounced in patients with sarcomatoid or mixed histology (HR for OS of $0.82(0.64-1.06)$ for epithelioid and $0.64(0.40-1.02)$ for sarcomatoid). However, another two trials with antiangiogenic have failed in demonstrate benefit in patients with mesothelioma. The phase II LUME/Meso designed to assess the efficacy of nintedanib plus chemotherapy, demonstrated evident benefit in epithelioid histology, but not in biphasic, however the number of patients with biphasic histology was too low to provide a reliable estimate of the treatment effect [24]. Also, the addition of cediranib to chemotherapy improved PFS and there was no difference in the effect of treatment by histological subtypes [25]. In the STELLAR trial TTFields delivery system in combination with chemotherapy for first line leads to a median OS and PFS longer than historical control [5]. In a post-hoc analysis, OS and PFS were longer in patients with epithelioid histology than in patients with other subtypes (OS 21.2 vs 12.1 months and PFS 8.3 vs 6.5 months, respectively).

Less evidence of the difference in the efficacy of treatment according to histology has been evaluated in studies of previously treated patients. Vinorelbine has shown clinical activity in a phase II study and responses were observed in all three histologic subtypes of mesothelioma, including those with sarcomatoid and biphasic tumors [26]. More recently, lurbinectedin demonstrated no significant differences in PFS and OS concerning the impact of histology, suggesting that lurbinectedin is likely to equalize the prognosis of the mesothelioma subtypes [27].

The impact of immunotherapy in mesothelioma has been recently demonstrated. Initial studies with monotherapy suggested efficacy, but randomized trials in previously treated patients are controversial. However, in first line setting, a recent study demonstrated better outcomes for immunotherapy over chemotherapy. In these studies of immunotherapy, predictive factors of response have been more studied. Keynote 028 phase I trial, enrolled previously treated PD-L1 positive mesothelioma patients and showed $40 \%$ of clinical benefit for more than 6 months [10]. In the trial $72 \%$ of patients were epithelioid but no results according to histology subtypes were reported. In the INITIATE trial, a single arm phase 2 
trial of nivolumab plus ipilimumab, disease control rate at 12 weeks was achieved by $68 \%$ [8]. The study included $86 \%$ of patients with epithelioid subtype and the small number of tumors with nonepithelioid histology did not allow a meaningful comparison between histological subtypes. MAPS2 trial also evaluated the addition of ipilimumab to nivolumab and demonstrated better outcomes for the combination [14]. Patients were stratified by histology with $85 \%$ of patients being epithelioid and responses were reported in all histological groups. PROMISE-meso failed in demonstrate superiority of pembrolizumab over chemotherapy in relapsed mesothelioma [11]. In this trial patients with nonepithelioid tumors had a non-significant poorer PFS and OS for pembrolizumab as compared to epithelioid. Two studies testing the combination of chemotherapy plus durvalumab have been reported. The Australian DREAM trial reported a 6 months PFS of $31 \%$ [28]. In the trial, $83 \%$ of patients were epithelioid and in a post-hoc analysis, responses were observed in all histological subtypes. The US PrE0505 trial also reported median OS of 20.4 months and this trial included $74.5 \%$ of patients with epithelioid tumors, but no data about the efficacy of the treatment by histology were reported [29].

Since the publication of the CheckMate 743 pointing differences in efficacy of chemotherapy according to histology, we sought to perform a retrospective analysis of the efficacy of the chemotherapy with histology at our institution. We evaluated 189 patients and we found, in agreement with other series that histology is a strong prognostic factor with a difference in median OS of 11.7 months (21.3 months in epithelioid versus 9.6 months in nonepithelioid). In our real-world series, we could demonstrate that histology is a prognostic factor for PFS in favor of epithelioid histology in patients treated with chemotherapy with a median PFS of 4.8 versus 3.6 months $(p=0.03)$. We detected that patients with epithelioid histology treated with cisplatin had higher benefit than patients treated with carboplatin (4.5 months versus 3.6 months for patients treated with carboplatin). Despite the numerically higher PFS in patients with epithelioid tumors treated with cisplatin, there was no significant interaction between platinum agent and histology in Cox models, suggesting that histology is not a determinant of platinum agent sensitivity.

At the moment, the trials that led approval of pemetrexed and raltitrexed in malignant mesothelioma did not reported difference in efficacy of the chemotherapy according to histology, and the addition of bevacizumab demonstrated efficacy of treatment in all subgroups with better outcomes for sarcomatoid and biphasic tumors. Only the combination of carboplatin plus pemetrexed reported no responses in sarcomatoid mesothelioma. Our results, in accordance with previous studies confirms that histology is a prognostic factor. In our series epithelioid histology was a significant determinant of PFS in patients treated with chemotherapy, confirming one of the conclusions of the CheckMate 743 pointing worse efficacy of chemotherapy in nonepithelioid patients. In our study we included a small cohort of patients treated with immunotherapy (27 patients) and in this cohort we did not detect differences in PFS according to histology.

Our results have some limitations. This is a real-world series including all patients treated at one single institution. In our series, the number of epithelioid histology patients was high (76\%) but this percentage 
is in line with the proportion of epithelioid patients included in clinical trials. We compare the impact of the treatment by histology but the number of patients with no epithelioid included is small.

In conclusion, in our series we confirm that histology is a prognostic factor and patients with nonepithelioid tumors had worse survival. Patients with epithelioid histology presented better PFS than patients with nonepithelioid tumors. Ongoing studies combining checkpoint inhibitors plus chemotherapy are evaluating the impact of histology in the outcomes.

\section{Declarations}

\section{Competing interest}

S.C. reports advisory role and/or travel compensation: Bristol-Myers Squibb Recipient F, Hoffmann La Roche AG, Pfizer, Boehringer Ingelheim, MSD Oncology, Amphera. P.I. reports advisory role and/or travel compensation: Bristol-Myers Squibb Recipient, F. Hoffmann, La Roche AG, Merck Sharp \& Dohme, Boehringer Ingelheim, MSD Oncology, Rovi, Kyowa Kirin, Grunenthal Pharma S.A., Pfizer, Medscape, Kern Pharma. A.C. reports advisory role and/or travel compensation: Bristol-Myers Squibb Recipient, $F$. Hoffmann La Roche AG, Pfizer, Boehringer Ingelheim, MSD Oncology, Kyowa Kirin, Celgene, Leo Pharma, Medscape, Kern Pharma. N.P. reports advisory role and/or travel compensation: MSD Oncology, Merck Sharp and Dohme, Bristol-Myers Squibb Recipient, F. Hoffmann La Roche AG, Pfizer, Boehringer Ingelheim, Grunenthal Pharma, S.A, Kern Pharma. A.N. reports advisory role, speaker's bureau or travel compensation: Bristol-Myers Squibb, F. Hoffmann La Roche AG, Pfizer, Boehringer Ingelheim, Oryzon Genomics, Oryzon Genomics, Astra Zeneca. A.M.M. provided consultation, attended advisory boards and/or speaker's bureau for the following organizations: BMS, Roche, MSD, Pfizer, Boehringer Ingelheim, AstraZeneca. C.C. and J.F. had been partially supported by Grant for Oncology EMD Serono research funding to EF. R.D. advisory role at Roche, Boehringer Ingelheim, has received a speaker's fee from Roche, Ipsen, Amgen, Servier, Sanofi, Merck Sharp \& Dohme, research grant from Merck and Pierre Fabre. E.F. reports the following conflicts of interest: advisory role or speaker's bureau: AbbVie, AstraZeneca, BerGenBio, Beigene, Bayer, Blueprint medicines, Boehringer Ingelheim, Bristol-Meyers Squibb, Celgene, Eli Lilly, Glaxo smith kline, Guardant Health, Janssen, Medscape, Merck KGaA, Merck Sharp \& Dohme, Merck Serono, Novartis, Pfizer, priME Oncology, Peptomyc, Peervoice, Puma, Regeneron, Sanofi, Syneos Health, Springer, Roche, Samsung, Takeda, Touchtime. Board: Grifols, independent member. Research funding: Fundación Merck Salud, Grant for Oncology Innovation EMD Serono. C.C. and J.F. had been partially supported by Grant for Oncology EMD Serono research funding to EF. All remaining authors have declared no conflicts of interest.

\section{Authors contribution}

All authors contributed to the design of the study, the statistical analysis, reviewed the manuscript during the writing process and approved the final version to be published

\section{Acknowledgments}


We thank our patients and their families for accepting to participate in this study.

\section{References}

1. Selikoff IJ, Hammond EC, Seidman H. Latency of asbestos disease among insulation workers in the United States and Canada. Cancer. 1980 Dec 15;46(12):2736-40. doi: 10.1002/10970142(19801215)46:12<2736::aid-cncr2820461233>3.0.co;2-I. PMID: 7448712.

2. Alpert N, van Gerwen $M$, Taioli E. Epidemiology of mesothelioma in the $21^{\text {st }}$ century in Europe and the United States, 40 years after restricted/banned asbestos use. Transl Lung Cancer Res. 2020 Feb;9(Suppl 1):S28-S38. doi: 10.21037/tlcr.2019.11.11. PMID: 32206568; PMCID: PMC7082259.

3. Vogelzang NJ, Rusthoven JJ, Symanowski J, Denham C, Kaukel E, Ruffie P, Gatzemeier U, Boyer M, Emri S, Manegold C, Niyikiza C, Paoletti P. Phase III study of pemetrexed in combination with cisplatin versus cisplatin alone in patients with malignant pleural mesothelioma. J Clin Oncol. 2003 Jul 15;21(14):2636-44. doi: 10.1200/JC0.2003.11.136. PMID: 12860938

4. Zalcman G, Mazieres J, Margery J, Greillier L, Audigier-Valette C, Moro-Sibilot D, Molinier O, Corre R, Monnet I, Gounant V, Rivière F, Janicot H, Gervais R, Locher C, Milleron B, Tran Q, Lebitasy MP, Morin F, Creveuil C, Parienti JJ, Scherpereel A; French Cooperative Thoracic Intergroup (IFCT). Bevacizumab for newly diagnosed pleural mesothelioma in the Mesothelioma Avastin Cisplatin Pemetrexed Study (MAPS): a randomised, controlled, open-label, phase 3 trial. Lancet. 2016 Apr 2;387(10026):14051414. doi: 10.1016/S0140-6736(15)01238-6. Epub 2015 Dec 21. Erratum in: Lancet. 2016 Apr 2;387(10026):e24. PMID: 26719230.

5. Ceresoli GL, Aerts JG, Dziadziuszko R, Ramlau R, Cedres S, van Meerbeeck JP, Mencoboni M, Planchard D, Chella A, Crinò L, Krzakowski M, Rüssel J, Maconi A, Gianoncelli L, Grosso F. Tumour Treating Fields in combination with pemetrexed and cisplatin or carboplatin as first-line treatment for unresectable malignant pleural mesothelioma (STELLAR): a multicentre, single-arm phase 2 trial. Lancet Oncol. 2019 Dec;20(12):1702-1709. doi: 10.1016/S1470-2045(19)30532-7. Epub 2019 Oct 15. Erratum in: Lancet Oncol. 2020 Feb;21(2):e70. PMID: 31628016.

6. Ceresoli GL, Zucali PA, Favaretto AG, Grossi F, Bidoli P, Del Conte G, Ceribelli A, Bearz A, Morenghi E, Cavina R, Marangolo M, Parra HJ, Santoro A. Phase II study of pemetrexed plus carboplatin in malignant pleural mesothelioma. J Clin Oncol. 2006 Mar 20;24(9):1443-8. doi: 10.1200/JCO.2005.04.3190. PMID: 16549838.

7. Castagneto B, Botta M, Aitini E, Spigno F, Degiovanni D, Alabiso O, Serra M, Muzio A, Carbone R, Buosi R, Galbusera V, Piccolini E, Giaretto L, Rebella L, Mencoboni M. Phase II study of pemetrexed in combination with carboplatin in patients with malignant pleural mesothelioma (MPM). Ann Oncol. 2008 Feb;19(2):370-3. doi: 10.1093/annonc/mdm501. Epub 2007 Dec 20. PMID: 18156144

8. Disselhorst MJ, Quispel-Janssen J, Lalezari F, Monkhorst K, de Vries JF, van der Noort V, Harms E, Burgers S, Baas P. Ipilimumab and nivolumab in the treatment of recurrent malignant pleural mesothelioma (INITIATE): results of a prospective, single-arm, phase 2 trial. Lancet Respir Med. 2019 Mar;7(3):260-270. doi: 10.1016/S2213-2600(18)30420-X. Epub 2019 Jan 16. PMID: 30660511. 
9. Okada M, Kijima T, Aoe K, Kato T, Fujimoto N, Nakagawa K, Takeda Y, Hida T, Kanai K, Imamura F, Oizumi S, Takahashi T, Takenoyama M, Tanaka H, Hirano J, Namba Y, Ohe Y. Clinical Efficacy and Safety of Nivolumab: Results of a Multicenter, Open-label, Single-arm, Japanese Phase II study in Malignant Pleural Mesothelioma (MERIT). Clin Cancer Res. 2019 Sep 15;25(18):5485-5492. doi: 10.1158/1078-0432.CCR-19-0103. Epub 2019 Jun 4. PMID: 31164373.

10. Alley EW, Lopez J, Santoro A, Morosky A, Saraf S, Piperdi B, van Brummelen E. Clinical safety and activity of pembrolizumab in patients with malignant pleural mesothelioma (KEYNOTE-028): preliminary results from a non-randomised, open-label, phase 1b trial. Lancet Oncol. 2017 May;18(5):623-630. doi: 10.1016/S1470-2045(17)30169-9. Epub 2017 Mar 11. PMID: 28291584.

11. Popat S, Curioni-Fontecedro A, Dafni U, Shah R, O'Brien M, Pope A, Fisher P, Spicer J, Roy A, Gilligan D, Gautschi O, Nadal E, Janthur WD, López Castro R, García Campelo R, Rusakiewicz S, Letovanec I, Polydoropoulou V, Roschitzki-Voser H, Ruepp B, Gasca-Ruchti A, Peters S, Stahel RA. A multicentre randomised phase III trial comparing pembrolizumab versus single-agent chemotherapy for advanced pre-treated malignant pleural mesothelioma: the European Thoracic Oncology Platform (ETOP 9-15) PROMISE-meso trial. Ann Oncol. 2020 Dec;31(12):1734-1745. doi:

10.1016/j.annonc.2020.09.009. Epub 2020 Sep 22. PMID: 32976938.

12. Maio M, Scherpereel A, Calabrò L, Aerts J, Perez SC, Bearz A, Nackaerts K, Fennell DA, Kowalski D, Tsao AS, Taylor P, Grosso F, Antonia SJ, Nowak AK, Taboada M, Puglisi M, Stockman PK, Kindler HL. Tremelimumab as second-line or third-line treatment in relapsed malignant mesothelioma (DETERMINE): a multicentre, international, randomised, double-blind, placebo-controlled phase $2 \mathrm{~b}$ trial. Lancet Oncol. 2017 Sep;18(9):1261-1273. doi: 10.1016/S1470-2045(17)30446-1. Epub 2017 Jul 17. PMID: 28729154.

13. Fennell D, Ottensmeier C, Califano R, Hanna G, Ewings S, Hill K, Wilding S, et al. PS01.11 Nivolumab versus placebo in relapsed malignant mesothelioma: The CONFIRM phase 3 trial. IASLC 2929 Wordl Conference on Lung Cancer, Singapore, Worldwide Virtual Event

14. Scherpereel A, Mazieres J, Greillier L, Lantuejoul S, Dô P, Bylicki O, Monnet I, Corre R, Audigier-Valette C, Locatelli-Sanchez M, Molinier O, Guisier F, Urban T, Ligeza-Poisson C, Planchard D, Amour E, Morin F, Moro-Sibilot D, Zalcman G; French Cooperative Thoracic Intergroup. Nivolumab or nivolumab plus ipilimumab in patients with relapsed malignant pleural mesothelioma (IFCT-1501 MAPS2): a multicentre, open-label, randomised, non-comparative, phase 2 trial. Lancet Oncol. 2019 Feb;20(2):239-253. doi: 10.1016/S1470-2045(18)30765-4. Epub 2019 Jan 16. Erratum in: Lancet Oncol. 2019 Mar;20(3):e132. PMID: 30660609.

15. Baas P, Scherpereel A, Nowak AK, Fujimoto N, Peters S, Tsao AS, Mansfield AS, Popat S, Jahan T, Antonia S, Oulkhouir Y, Bautista Y, Cornelissen R, Greillier L, Grossi F, Kowalski D, Rodríguez-Cid J, Aanur P, Oukessou A, Baudelet C, Zalcman G. First-line nivolumab plus ipilimumab in unresectable malignant pleural mesothelioma (CheckMate 743): a multicentre, randomised, open-label, phase 3 trial. Lancet. 2021 Jan 21:S0140-6736(20)32714-8. doi: 10.1016/S0140-6736(20)32714-8. Epub ahead of print. PMID: 33485464. 
16. Nicholson AG, Sauter JL, Nowak AK, et al. EURACAN/IASLC proposals for updating the histologic classification of pleural mesothelioma: towards a more multidisciplinary approach. J Thorac Oncol. 2020;15(1):29-49.

17. Rami-Porta R. Staging Manual in Thoracic Oncology from Inetrnational Association for the study of Lung Cancer. ISBN:978-0-9832958-4-6

18. M Schemper and TL Smith. A note on quantifying follow-up in studies of failure time. Controlled clinical trials (1996) vol. 17 (4) pp. 343-346

19. Curran D, Sahmoud T, Therasse P, van Meerbeeck J, Postmus PE, Giaccone G. Prognostic factors in patients with pleural mesothelioma: the European Organization for Research and Treatment of Cancer experience. J Clin Oncol. 1998 Jan;16(1):145-52. doi: 10.1200/JC0.1998.16.1.145. PMID: 9440736.

20. Herndon JE, Green MR, Chahinian AP, Corson JM, Suzuki Y, Vogelzang NJ. Factors predictive of survival among 337 patients with mesothelioma treated between 1984 and 1994 by the Cancer and Leukemia Group B. Chest. 1998 Mar;113(3):723-31. doi: 10.1378/chest.113.3.723. PMID: 9515850.

21. Billé A, Krug LM, Woo KM, Rusch VW, Zauderer MG. Contemporary Analysis of Prognostic Factors in Patients with Unresectable Malignant Pleural Mesothelioma. J Thorac Oncol. 2016 Feb;11(2):249-55. doi: 10.1016/j.jtho.2015.10.003. Epub 2015 Dec 10. PMID: 26845118; PMCID: PMC491728

22. .Jänne PA, Wozniak AJ, Belani CP, Keohan ML, Ross HJ, Polikoff JA, Mintzer DM, Taylor L, Ashland J, Ye Z, Monberg MJ, Obasaju CK. Open-label study of pemetrexed alone or in combination with cisplatin for the treatment of patients with peritoneal mesothelioma: outcomes of an expanded access program. Clin Lung Cancer. 2005 Jul;7(1):40-6. doi: 10.3816/CLC.2005.n.020. PMID: 16098243.

23. van Meerbeeck JP, Gaafar R, Manegold C, Van Klaveren RJ, Van Marck EA, Vincent M, Legrand C, Bottomley A, Debruyne C, Giaccone G; European Organisation for Research and Treatment of Cancer Lung Cancer Group; National Cancer Institute of Canada. Randomized phase III study of cisplatin with or without raltitrexed in patients with malignant pleural mesothelioma: an intergroup study of the European Organisation for Research and Treatment of Cancer Lung Cancer Group and the National Cancer Institute of Canada. J Clin Oncol. 2005 Oct 1;23(28):6881-9. doi: 10.1200/JC0.20005.14.589. PMID: 16192580.

24. Grosso F, Steele N, Novello S, Nowak AK, Popat S, Greillier L, John T, Leighl NB, Reck M, Taylor P, Planchard D, Sørensen JB, Socinski MA, von Wangenheim U, Loembé AB, Barrueco J, Morsli N, Scagliotti G. Nintedanib Plus Pemetrexed/Cisplatin in Patients With Malignant Pleural Mesothelioma: Phase II Results From the Randomized, Placebo-Controlled LUME-Meso Trial. J Clin Oncol. 2017 Nov 1;35(31):3591-3600. doi: 10.1200/JC0.2017.72.9012. Epub 2017 Sep 11. PMID: 28892431.

25. Tsao AS, Miao J, Wistuba II, Vogelzang NJ, Heymach JV, Fossella FV, Lu C, Velasco MR, Box-Noriega B, Hueftle JG, Gadgeel S, Redman MW, Gandara DR, Kelly K. Phase II Trial of Cediranib in Combination With Cisplatin and Pemetrexed in Chemotherapy-Naïve Patients With Unresectable 
Malignant Pleural Mesothelioma (SWOG S0905). J Clin Oncol. 2019 Oct 1;37(28):2537-2547. doi: 10.1200/JC0.19.00269. Epub 2019 Aug 6. PMID: 31386610; PMCID: PMC7001793.

26. Steele JP, Shamash J, Evans MT, Gower NH, Tischkowitz MD, Rudd RM. Phase II study of vinorelbine in patients with malignant pleural mesothelioma. J Clin Oncol. 2000 Dec 1;18(23):3912-7. doi: 10.1200/JCO.2000.18.23.3912. PMID: 11099320.

27. Metaxas Y, Früh M, Eboulet El, Grosso F, Pless M, Zucali PA, Ceresoli GL, Mark M, Schneider M, Maconi A, Perrino M, Biaggi-Rudolf C, Froesch P, Schmid S, Waibel C, Appenzeller C, Rauch D, von Moos R; Swiss Group for Clinical Cancer Research (SAKK). Lurbinectedin as second- or third-line palliative therapy in malignant pleural mesothelioma: an international, multi-centre, single-arm, phase II trial (SAKK 17/16). Ann Oncol. 2020 Apr;31(4):495-500. doi: 10.1016/j.annonc.2019.12.009. Epub 2020 Jan 16. PMID: 32085891.

28. Nowak AK, Lesterhuis WJ, Kok PS, Brown C, Hughes BG, Karikios DJ, John T, Kao SC, Leslie C, Cook AM, Pavlakis N, Briscoe K, O'Byrne KJ, Karapetis CS, Lam WS, Langford A, Yip S, Stockler MR. Durvalumab with first-line chemotherapy in previously untreated malignant pleural mesothelioma (DREAM): a multicentre, single-arm, phase 2 trial with a safety run-in. Lancet Oncol. 2020 Sep;21(9):1213-1223. doi: 10.1016/S1470-2045(20)30462-9. PMID: 32888453.

29. Patrick M. Forde, Zhuoxin Sun, Valsamo Anagnostou, Hedy L. Kindler, William T. Purcell, Bernardo H. L. Goulart, Arkadiusz Z. Dudek, Hossein Borghaei, Julie R. Brahmer, Suresh S. Ramalingam. PrE0505: phase II multicenter study of anti-PD-L1, durvalumab, in combination with cisplatin and pemetrexed for the first-line treatment of unresectable malignant pleural mesothelioma (MPM) - A PrECOG LLC study J Clin Oncol 38: 2020 (suppl; abstr 9003)

\section{Tables}

Table 1: Patients characteristics 


\begin{tabular}{|c|c|c|}
\hline \multicolumn{3}{|c|}{ BASELINE PATIENTS CHARACTERISTICS } \\
\hline Characteristic & Number & Percentage \\
\hline Median age & \multicolumn{2}{|c|}{68 y $(45-88)$} \\
\hline \multicolumn{3}{|l|}{ Gender } \\
\hline Males & 132 & 70 \\
\hline Females & 57 & 30 \\
\hline \multicolumn{3}{|l|}{ PS } \\
\hline 0 & 44 & 23 \\
\hline 1 & 131 & 69 \\
\hline 2 & 14 & 13 \\
\hline \multicolumn{3}{|l|}{ Asbestos } \\
\hline Yes & 141 & 74 \\
\hline No & 47 & 26 \\
\hline \multicolumn{3}{|l|}{ Histology } \\
\hline Epithelioid & 145 & 76 \\
\hline Non-epithelioid & 44 & 24 \\
\hline \multicolumn{3}{|l|}{ Stage } \\
\hline II & 39 & 21 \\
\hline III & 84 & 45 \\
\hline IV & 59 & 33 \\
\hline \multicolumn{3}{|l|}{ NLR } \\
\hline$<5$ & 109 & 58 \\
\hline$\geq 5$ & 62 & 33 \\
\hline \multicolumn{3}{|l|}{ First line chemotherapy } \\
\hline Yes & 161 & 85 \\
\hline No & 28 & 15 \\
\hline \multicolumn{3}{|l|}{ Type of chemotherapy } \\
\hline Cisplatin-pemetrexed & 102 & 66 \\
\hline Carboplatin-pemetrexed & 32 & 27 \\
\hline
\end{tabular}


Table 2 Multivariate analysis

\section{A. Epithelioid population $(n=129)$}

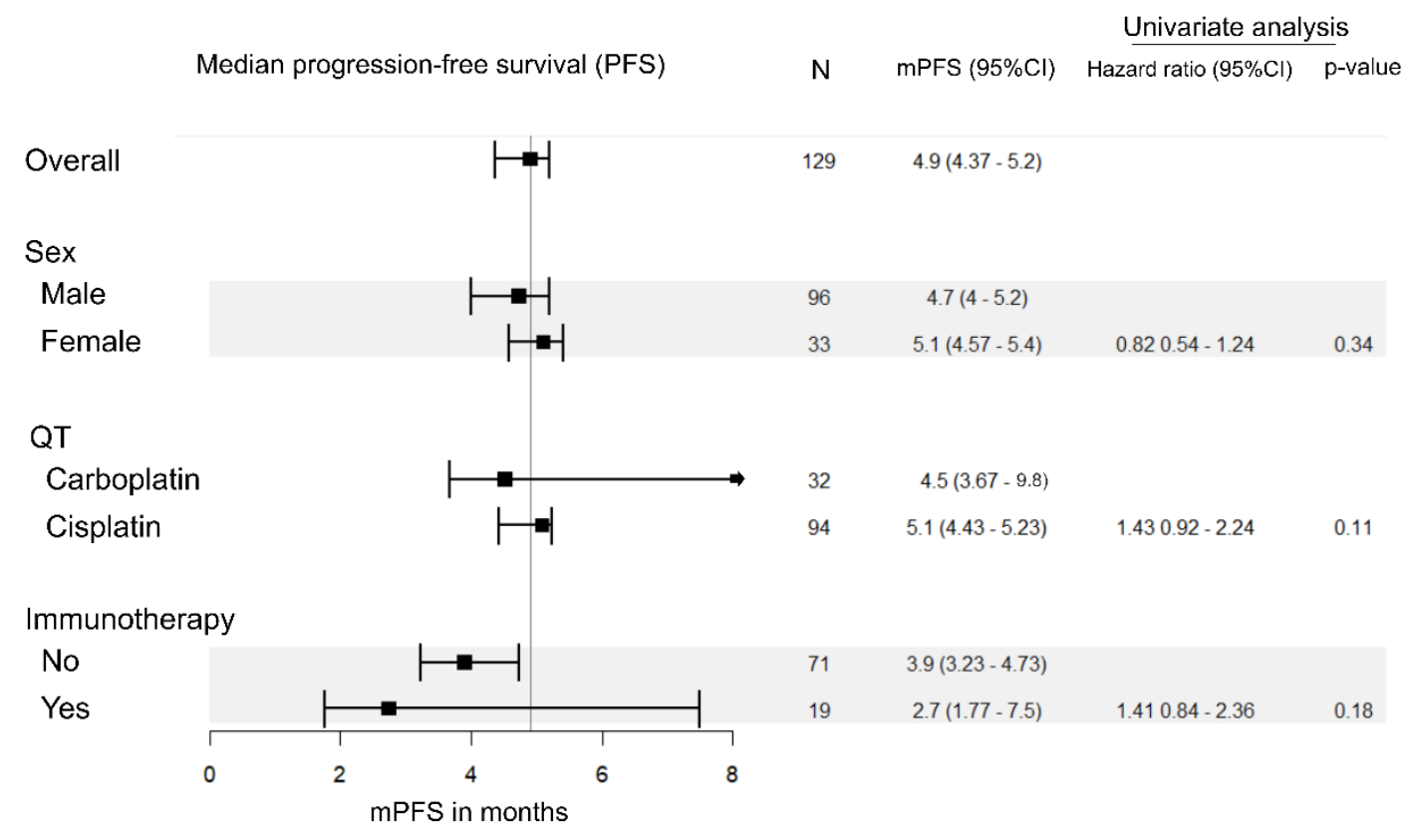

\section{B. No-Epithelioid population $(n=32)$}

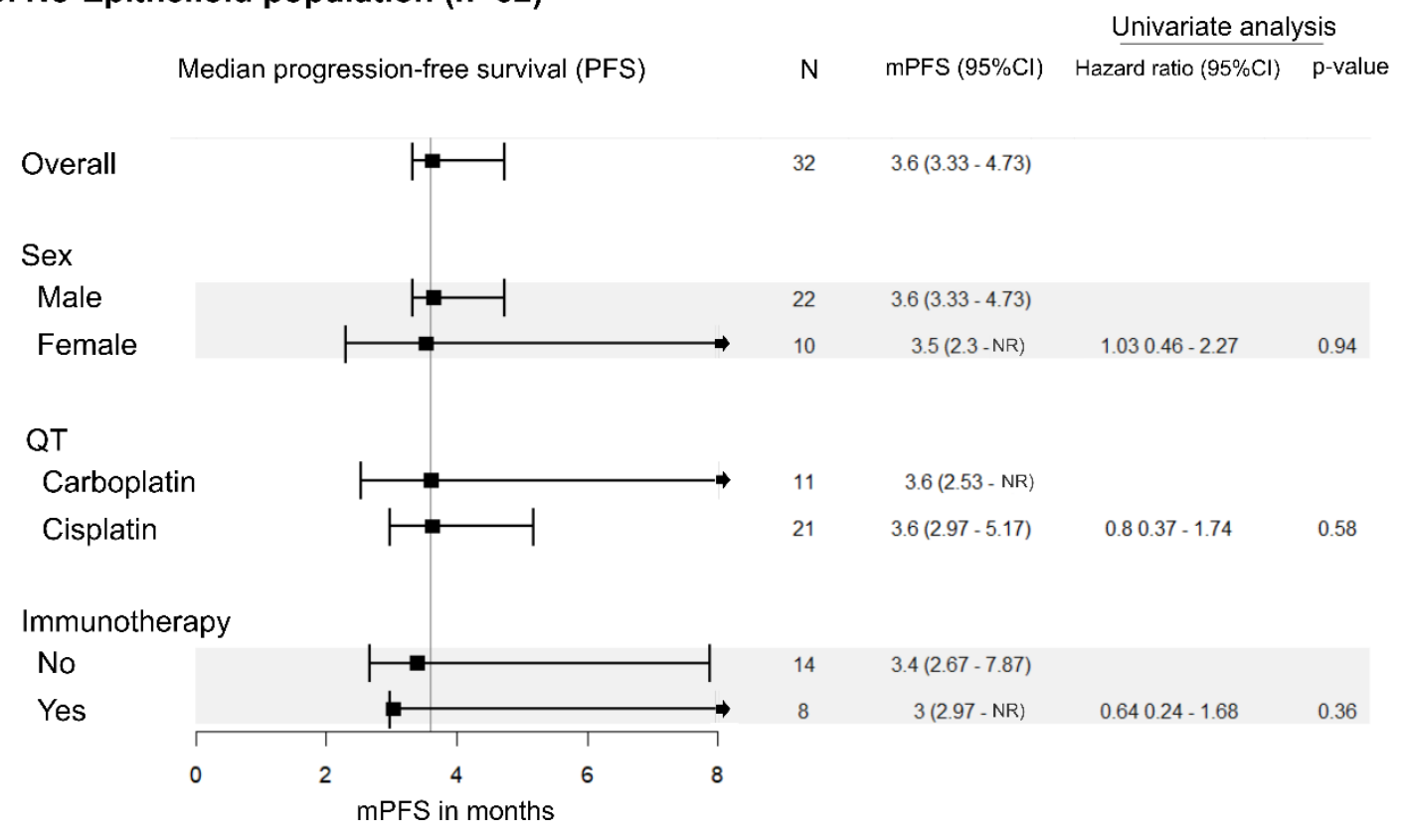

\section{Figures}


A
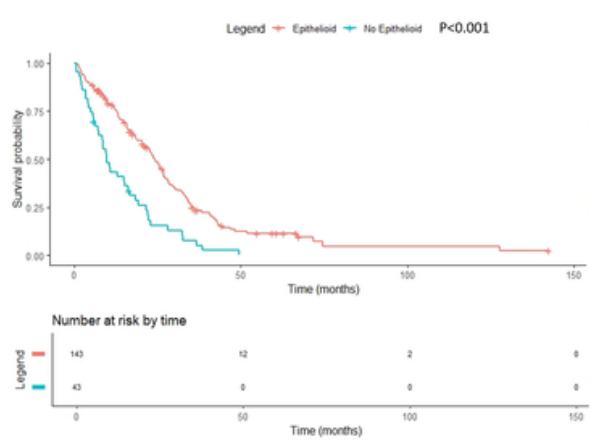

B

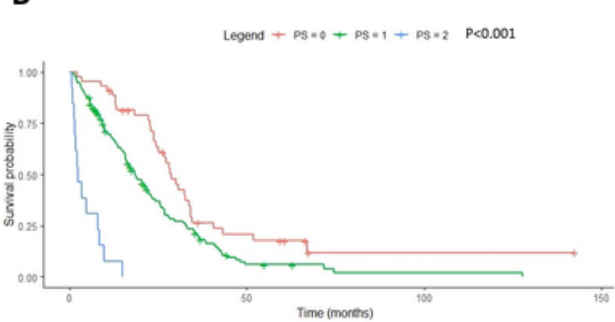

C

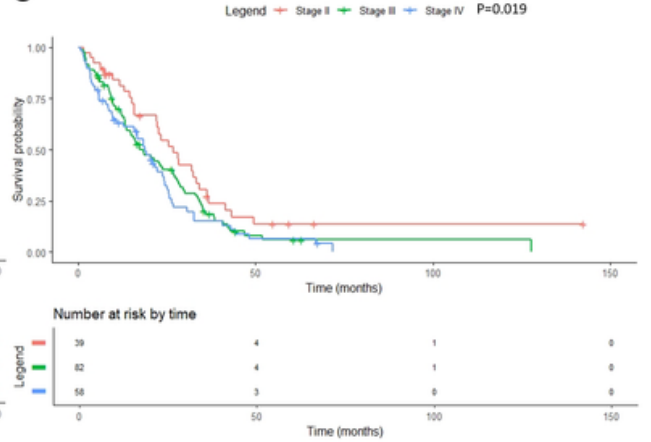

Figure 1

Kaplan-Meier overall survival according to histology (A), Performance status (B) and clinical stage (C)

A
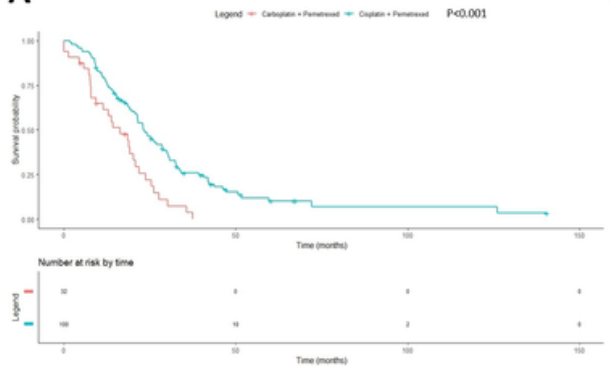

B

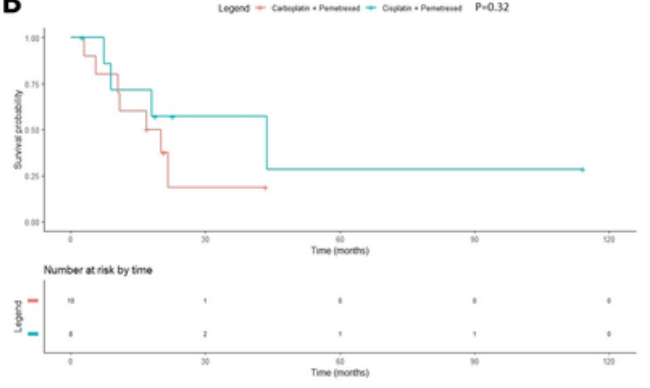

C

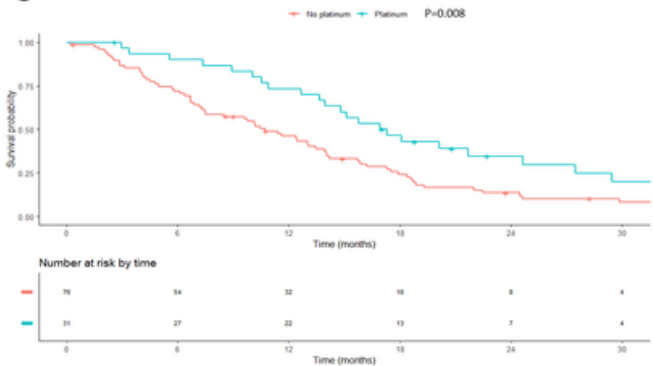

Figure 2

Kaplan-Meier overall survival according to type of systemic treatment: cisplatin versus carboplatin in first line(A), cisplatin versus carboplatin in second line (B) and platinum versus no platinum in second line (C) 
A

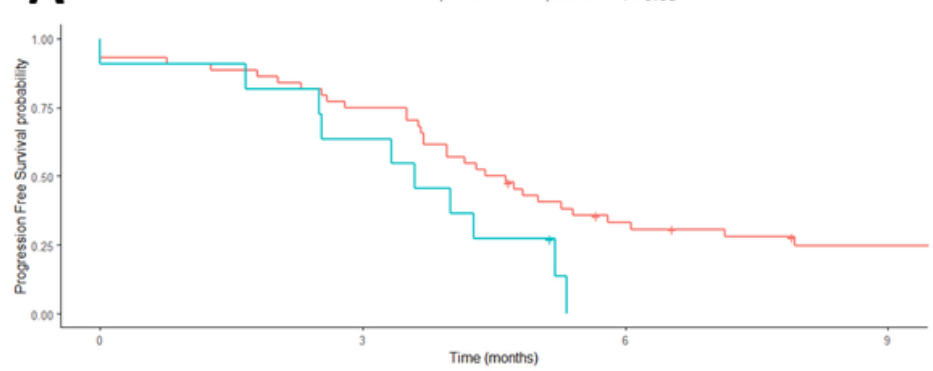

C

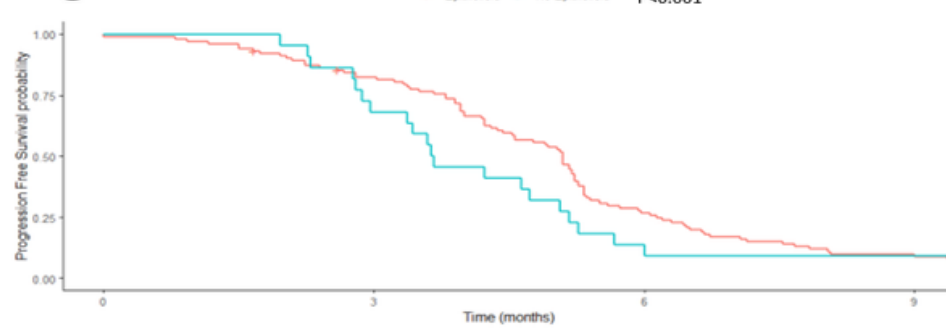

E

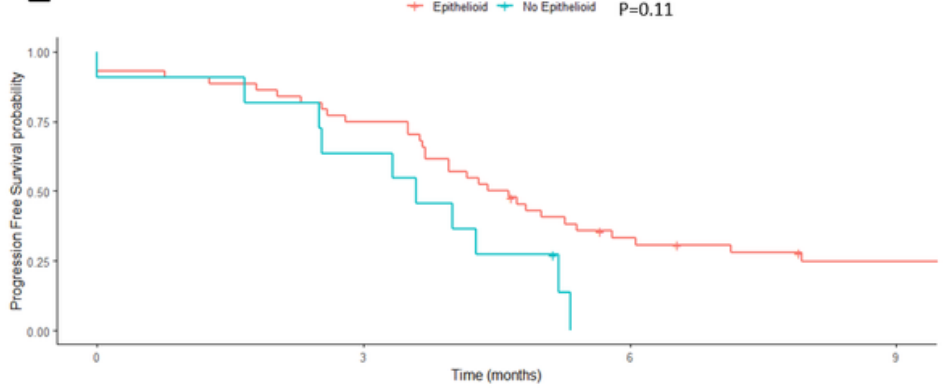

B

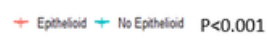

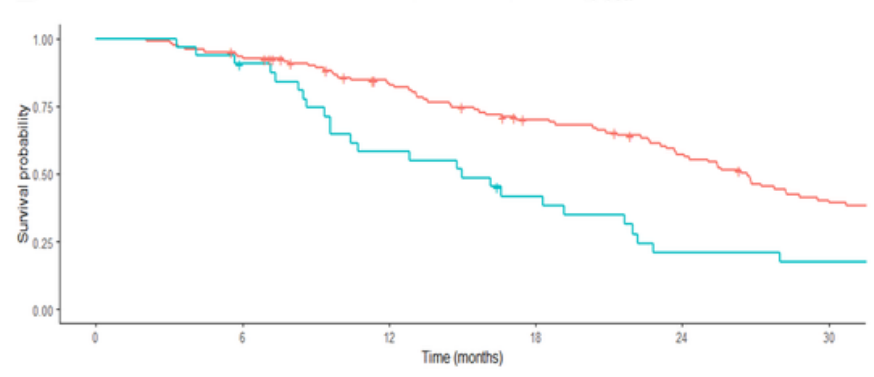

D

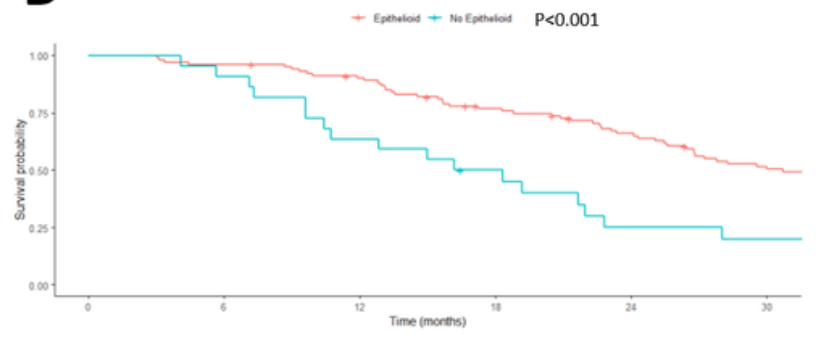

$\mathbf{F}$

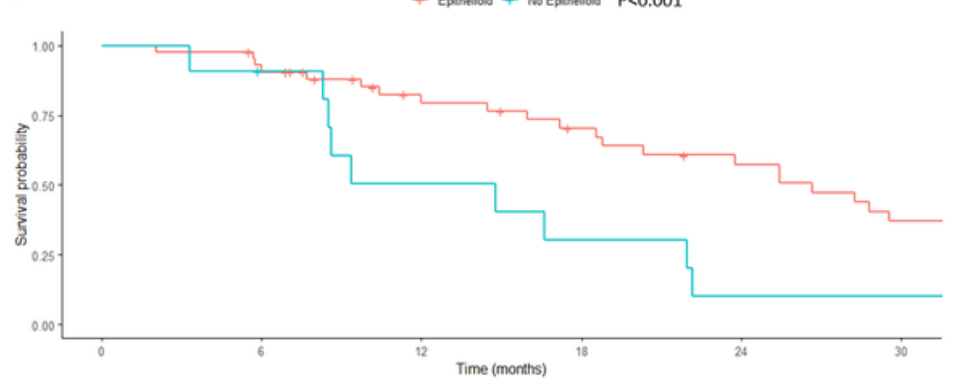

Figure 3

Kaplan-Meier overall survival according to hystology: PFS and OS of patients treated with first line chemotherapy $(A, B), P F S$ and OS of patients treated with cisplatin $(C, D)$ and PFS and OS of patients treated with carboplatin $(\mathrm{E}, \mathrm{F})$
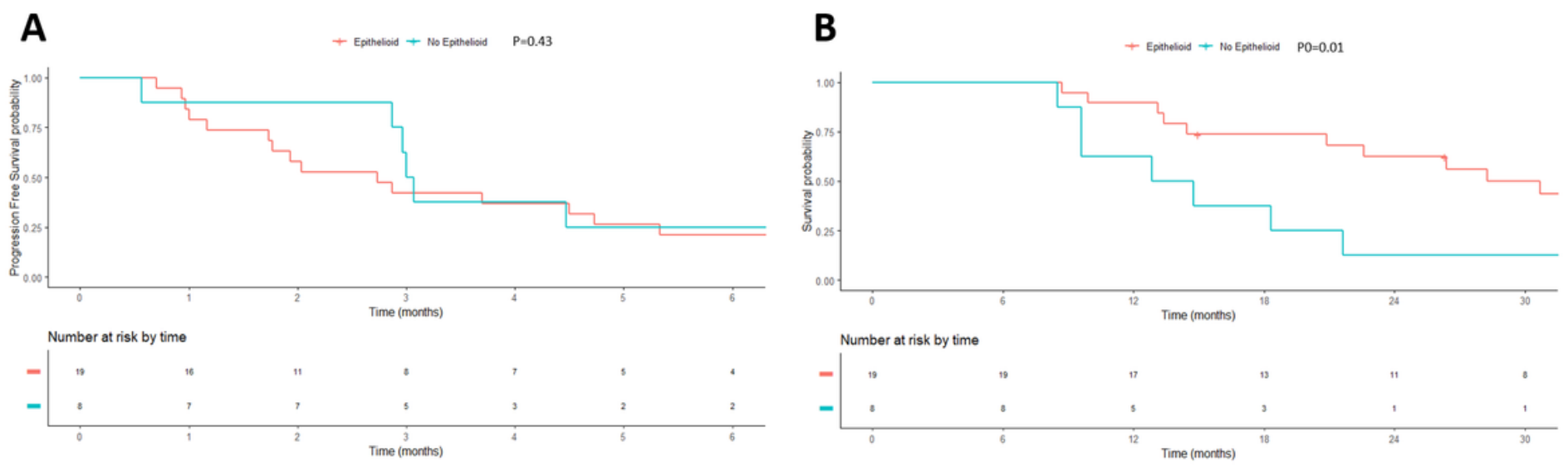

Figure 4 
Kaplan-Meier Survival according to histology in patients treated with cancer immunotherapy: PFS (A) and OS (B) 